\title{
Physicochemical Properties of Natural Based Products versus Synthetic Chemicals
}

\author{
Hilal Zaid ${ }^{1}$, Jamal Raiyn ${ }^{1}$, Ahmed Nasser ${ }^{2}$, Bashar Saad ${ }^{1}$ and Anwar Rayan ${ }^{1, *}$ \\ ${ }^{I}$ Drug Discovery Informatics Laboratory, QRC-Qasemi Research Center, Al-Qasemi Academic College, P.O.B. 124, \\ Baqa El-Gharbia 30100, Israel \\ ${ }^{2}$ Institute of Soils, Water and Environmental Sciences, ARO, Volcani Center, Bet Dagan 50-250, Israel
}

\begin{abstract}
The majority of the currently used cosmetics and drugs are natural products-based compounds or their derivatives. This could add weight to the argument that natural based products are inherently better tolerated in the body than synthetic chemicals and have higher chance to be approved as new drugs. The present study was undertaken to analyze a natural product database compared to synthetic chemicals and to search for discriminative physicochemical properties that may probably help in differentiating between natural and synthetic compounds. We have formulated rules to assess the natural likeness of chemicals and thereby discriminate between natural-based and synthetic chemicals. A Mathews Correlation Coefficient of 0.5 was obtained; nearly $81 \%$ of natural-based products and $68 \%$ of synthetic chemicals were precisely classified using this filter. The property criteria for drug-likeness and lead-likeness are more pronounced in natural products rather than synthetic ones. The fraction of synthetic chemicals which are natural-like could have higher chance to be successful drug.
\end{abstract}

Keywords: Natural product, natural-likeness, drug-likeness, in silico prediction model, chemo-informatics.

\section{INTRODUCTION}

Natural product-based medicines, particularly, herbalbased drugs represented about 80 percent of all drugs in use by 1990 [1-3]. They represented the main source of leads for the development of new drugs for centuries [4-6]. During the past couple of decades, after the introduction of high throughput synthesis and combinatorial chemistry, natural products became less significant source of drugs and leads. Although global expenditure on drug research has doubled since 1991, the number of new drug entities approved annually decreased by $50 \%$ or even more $[7,8]$. To change this situation, the players in the pharmaceutical industry shifted their interest back to natural or natural-based products [915]. It becomes commonly accepted that natural based products are inherently better tolerated in the body and have innate advantages for drug discovery and development over synthetic chemicals [16-19].

Although computational methods are well established in drug discovery and molecular design [20-22], their application in the field of natural products is still in its infancy. During the last decade we have seen an increased interest in the application of in silico tools in the natural productbased drug discovery in order to accelerate identification of bioactive natural-based products, maximize their efficacy and minimize potential side effects. Computer assisted approaches [23], such as docking [24-27], pharmacophore

*Address correspondence to this author at the Drug Discovery Informatics Laboratory, QRC-Qasemi Research Center, Al-Qasemi Academic College, P.O.B. 124, Baqa El-Gharbia 30100, Israel; Tel: + 972-4-6286761/4;

Fax: + 972-4-6286762; E-mail: a_rayan@qsm.ac.il modeling [28-30] and virtual screening [31-34] have been carried out and reported related to the field of bioactive natural products. In order to introduce the natural products features into the design of drug candidates, the discriminative features of natural products need to be unraveled. Most scientific reports utilized structural features and substructures for scoring natural likeness of products [35-38]. However, the utility of ranges-based filters which is composed of 2D physicochemical descriptors in modeling could give sometimes less discriminative models but guarantee finding new chemical entities in higher rates $[39,40]$. The present study aims to introduce a new highly efficient rules-based filter to assess the natural likeness of chemicals utilizing physicochemical properties and thereby differentiates between natural-based and synthetic chemicals.

\section{MATERIALS AND METHODS}

A natural products database of commercially available natural products and natural product derivatives was downloaded from ZINC database (ZINC natural products http:// zinc.docking.org/catalog/npd.in). Other twenty thousands synthetic compounds were selected from ZINC database of commercially available chemicals (http://zinc.docking.org/ subset1/). All selected synthetic chemical are drug like according to Lipinski rules of five and have entire diversity of Tanimoto < 0.7. In this study we seek a method that could provide simple rules to be utilized for differentiation between natural based and synthetic chemicals. For this purpose we have utilized 2D descriptors that were computed by MOE 2008.10. MOE is an integrated drug discovery software package with tools for chemoinformatics, bioinformat- 
ics, molecular modeling and visualization. It was developed by Chemical Computing Group, Inc. Montreal, Canada.

The decision, which set of relevant descriptors to use for differentiating between natural based and synthetic chemicals, is crucial. We sought after the most significant set of descriptors from which guidelines for natural likeness could be extracted. The selection has been performed automatically as following: all descriptors were evaluated separately and the best discriminative descriptor was chosen to be the core. The second descriptor to be added to the core was selected from the rest descriptors while giving the best performance in discrimination. The process continued until we have an efficient rules-of-thumb filter.

We aim to construct a filter consisting of ranges of few descriptors that can differentiate well between natural based and synthetic chemicals. For this purpose, descriptors' ranges were optimized simultaneously in exhaustive search, by maximizing a function (Matthews' Correlation Coefficient, MCC) [41] that considers each of the four possible outcomes for any chemical - Positive, Negative, False Positive and False Negative. Higher MCC means better distinction.

The division process of databases into training set and test set was performed by a random choice with $50 \%$ of the natural-based/ synthetic chemicals, while the remaining (50\% of the databases) was used as a test set.

The need for a combinatorial optimization of descriptors' ranges dictates the requirement to transform descriptor values into discrete ones. Some descriptors already have a discrete character, i.e., the numbers of Oxygen atoms, H-bond acceptors etc, while others, such as molecular weight, VDW surface area, etc., are continuous. The transformation to discrete character was limited to give 50 values for upper and lower limit ranges each.

A set of rules is constructed by picking lower limit and upper limit for each descriptor. Each set has two values for each descriptor, constituting the range which is considered to be the "correct" one (by that set of descriptors) for natural likeness. The "correctness" of this set is measured by its MCC value, described below. The constructed set of rules is applied to the natural based chemicals in the training sets to calculate the value of the scoring function, its Matthews Correlation Coefficient (MCC) (equation 1).

$$
M C C=\frac{(P N)-\left(P_{f} N_{f}\right)}{\sqrt{\left(N+N_{f}\right)\left(N+P_{f}\right)\left(P+N_{f}\right)\left(P+P_{f}\right)}}
$$

Where, $\mathrm{P}$ and $\mathrm{N}$ are the percentages of true positive and true negative predictions while $\mathrm{P}_{f}$ and $\mathrm{N}_{f}$ are the percentages of false positives and false negatives, respectively. True positives are natural based chemicals that are identified as natural-like chemicals. False positives are synthetic chemicals that are identified as natural-like chemicals. False negatives are natural based chemicals identified as synthetic-like chemicals, and true negatives are synthetic chemicals, identified as synthetic-like chemicals. The possible values for MCC range between -1.0 and 1.0 (1.0 for a perfect prediction and -1.0 for a completely erroneous prediction).
An exhaustive search is performed for all combinations (more than one hundred million options) and the resulting sets of rules are sorted based on their MCC score. The best set of rules is presented.

\section{RESULTS AND DISCUSSION}

Results obtained from the present study indicate that $98.4 \%$ of chemicals in the natural products database obey Lipinski rule of 5 [42] and 85.6\% obey Oprea lead-like rule [43]. An analysis of 2245 drugs were used in order to formulate the Lipinski rule of 5, which indicates that orally bio available drug-like molecules are likely to have $\leq 5 \mathrm{H}$-bond donors, $\leq 10$ H-bond acceptors, $\leq 500$ molecular weight and $\leq 5 \log$ P. However, an analysis of 96 drugs and leads from which they were derived, were utilized to extract the Oprea rules for lead likeness, stating that lead molecules are more likely to have $\leq 450$ molecular weight, between -3.5 and 4.5 $\log \mathrm{P}, \leq 4$ rings, $\leq 10$ non-terminal single bonds, $\leq 5$ hydrogen bond donors and $\leq 8$ hydrogen bond acceptors. Drug like or lead like molecules should have less than 2 violations descriptor's value that is out of the range. Fig. (2) shows the number of violation for drug-likeness and lead-likeness in natural products database. Fig. (2a) demonstrates that $98.8 \%$ of the compounds had less than 2 violations to Lipinski rule of 5, with $87.3 \%$ having no violation. Fig. (2b) shows that more than $85 \%$ of the natural products had less than 2 violations to Oprea lead-likeness, with $65.8 \%$ having no violation. These findings indicate that the property criteria for druglikeness and lead-likeness are highly pronounced in natural products. The distribution for the individual properties of the natural products is shown in Fig. (1). The molecular weight, H-bond donors, H-bond acceptors and $\log \mathrm{P}(\mathrm{o} / \mathrm{w})$ distributions peak at 300-400 dalton, 0,4 and 2-3 units of $\log \mathrm{P}$ respectively.

Bajorath and his co-workers [37] proposed several sets of descriptors for distinguishing between natural products and synthetic molecules. Four out of the six proposed models composed only of 2D physicochemical descriptors while the two others include structural elements. We have utilized those sets of descriptors as well as the four descriptors of Lipinski to construct a discriminative filter able to distinguish between natural and synthetic molecules by optimization ranges. Out of Bajorath proposed models, Model 5 (M5) composed of 8 descriptors gave the best result compared to the other sets in that group. As shown in Table 1, it has a Matthews' Correlation Coefficient of 0.36 , retaining $79 \%$ of the natural products and $56 \%$ of the synthetic molecules.

A new discriminative filter between natural products and synthetic chemicals was constructed as described in the method section. In order to ensure non-redundant information in the features utilized for model construction, correlation between descriptors in natural products database were computed by WEKA (http://www.cs.waikato.ac.nz/ml/ weka/) and stored into a matrix, see Table 2. The values of the correlation coefficient are constricted into the interval $[-1,1]$, correlation coefficient equal 1 corresponding to perfectly correlated features while coefficient of -1 corresponding to perfectly uncorrelated features. As shown in Table 2, 
Table 1. Filters for Differentiating between Natural Products and Synthetic Molecules

\begin{tabular}{|c|c|c|c|}
\hline Model & MCC & True Natural Products & True Synthetic Molecules \\
\hline \hline Lipinski descriptors $^{1}$ & 0.27 & 78 & 48 \\
\hline M1 $^{2}$ & 0.28 & 89 & 34 \\
\hline M2 $^{3}$ & 0 & 100 & 0 \\
\hline M3 $^{4}$ & 0.34 & 69 & 65 \\
\hline M5 $^{5}$ & 0.36 & $\mathbf{7 9}$ & 56 \\
\hline Our model $^{6}$ & $\underline{\mathbf{0 . 5}}$ & $\underline{\mathbf{8}}$ & $\underline{\mathbf{6}}$ \\
\hline
\end{tabular}

1: Molecular Weight, Hydrogen Bond Acceptors, Hydrogen Bond Donors and calculated $\log \mathrm{P}(\mathrm{o} / \mathrm{w})$.

2: petitjean, PEOE_VSA_+2, b_double, PEOE_VSA_-5, PEOE_VSA_+3, radius and vsa_other

3: PC+, PC-, RPC+, RPC-, Fcharge, a_nI, a_nP, a_nBr, b_triple, vsa_acid, vsa_base

4: b_1rotR, VadjEq, a_ICM, PEOE_RPC-, VdistEq, VdistMa, PEOE_RPC+ and VAdjMa

5: a_ICM, bpol, chi0v_C, b_double, chilv, a_nH, b_single and b_ar

6: KierFlex, a $\_$nN, chiral_u, KierFlex and vsa $\_$acid

a

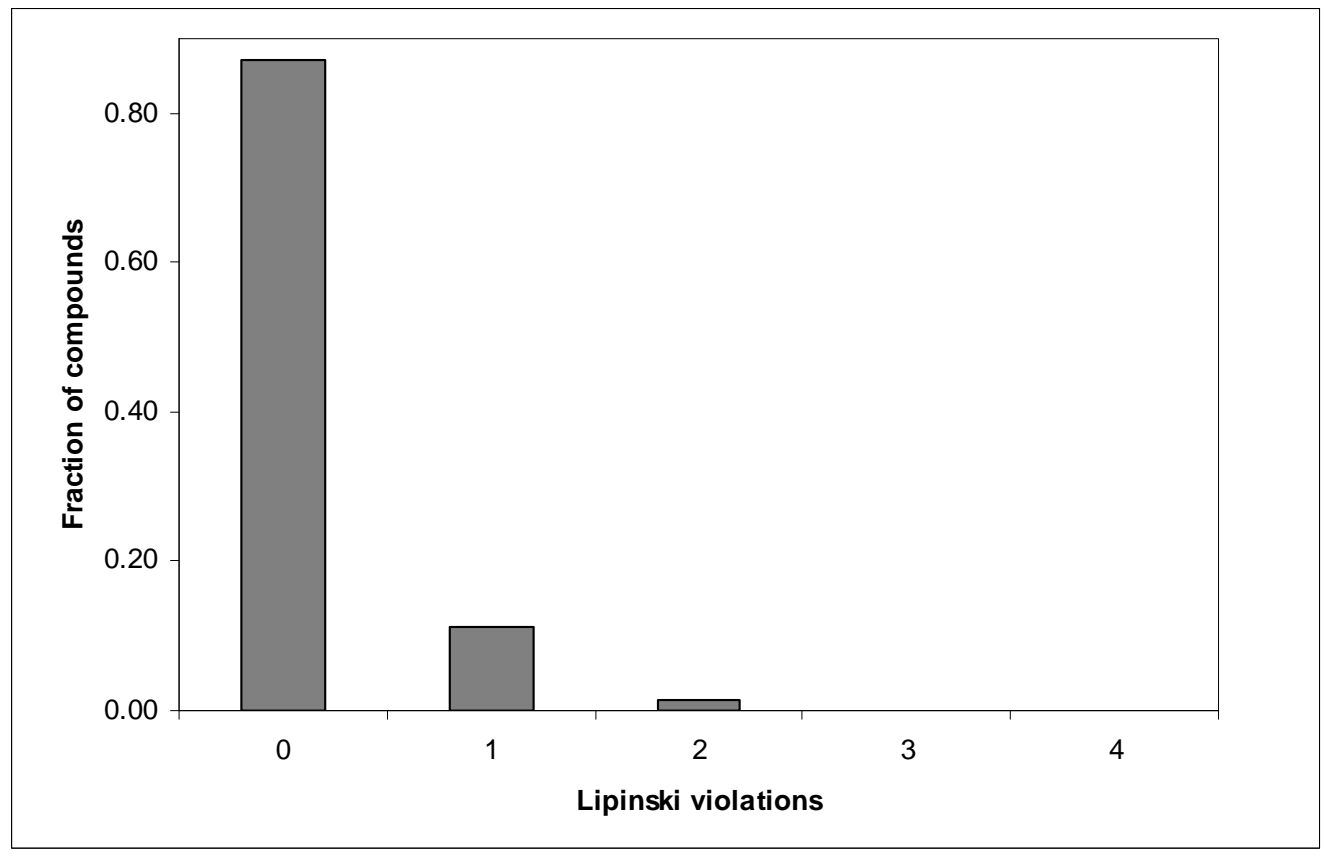

b

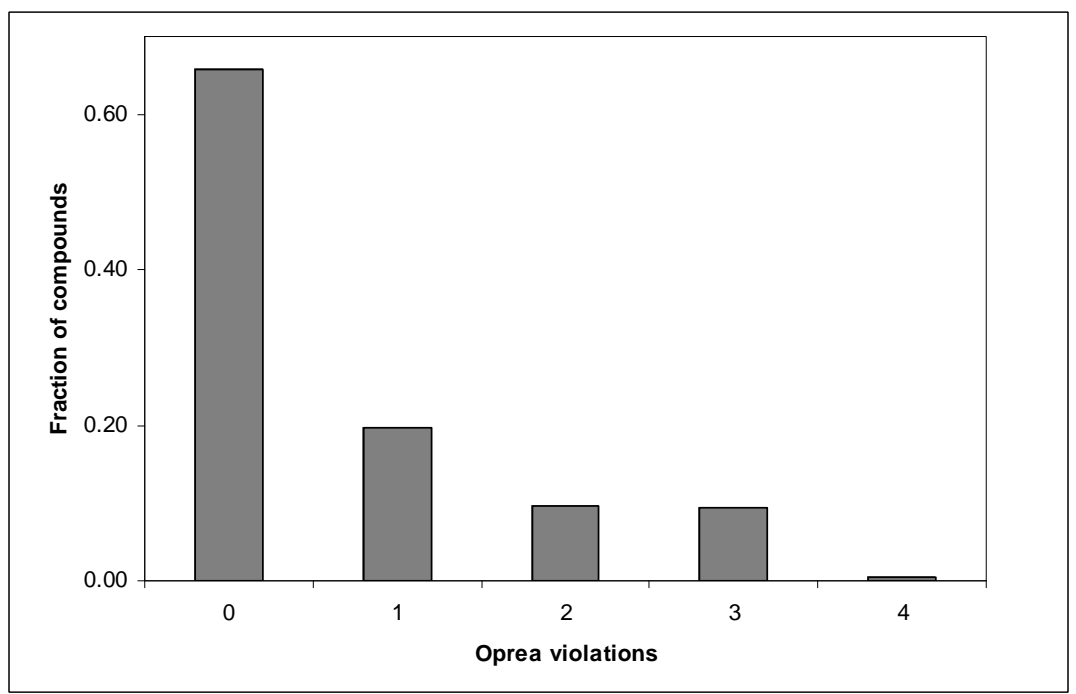

Fig. (1). Histograms of a) Lipinski violations for drug-likeness and b) Oprea violations for lead-likeness. Drug like or lead like could bear up to 1 violation. 
a

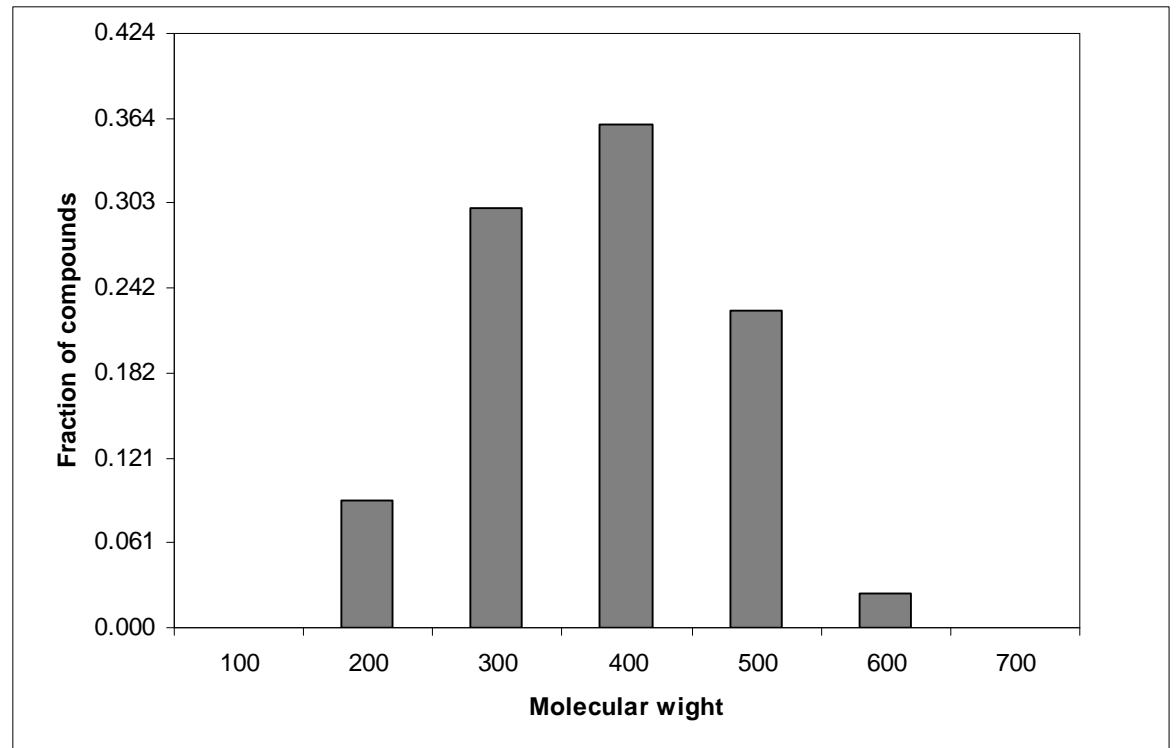

b

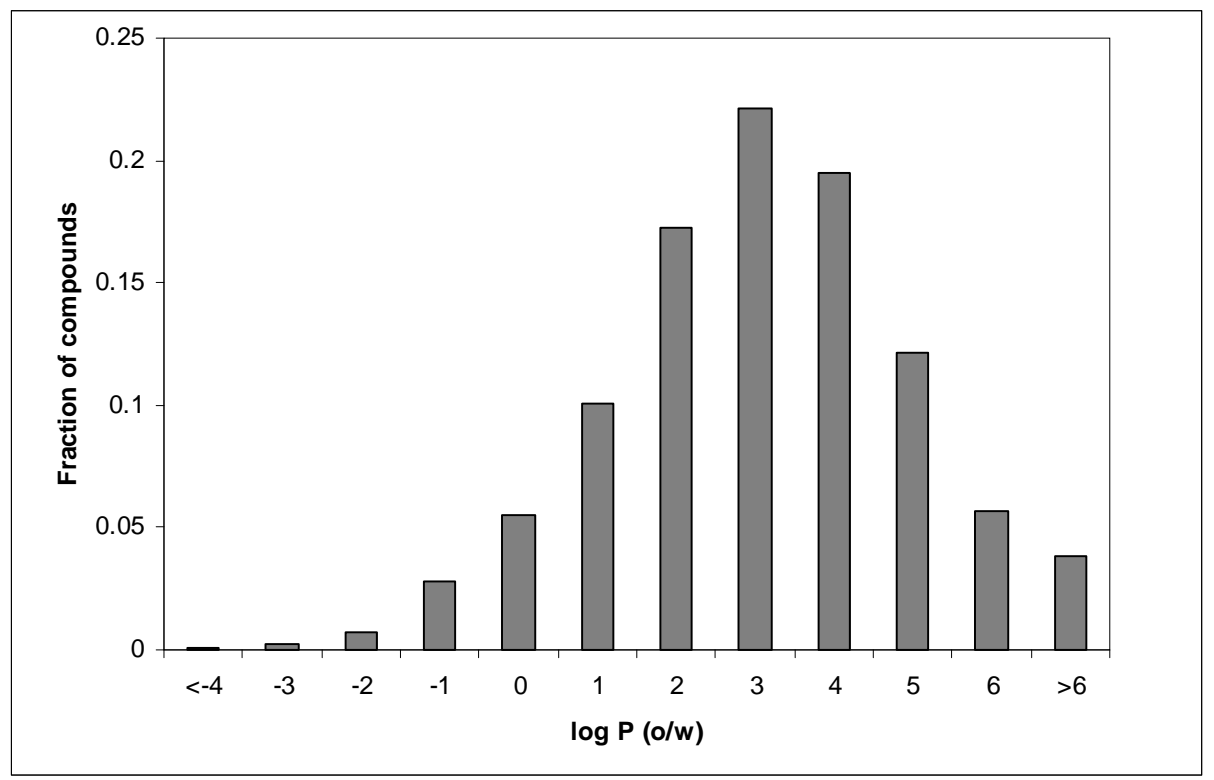

c

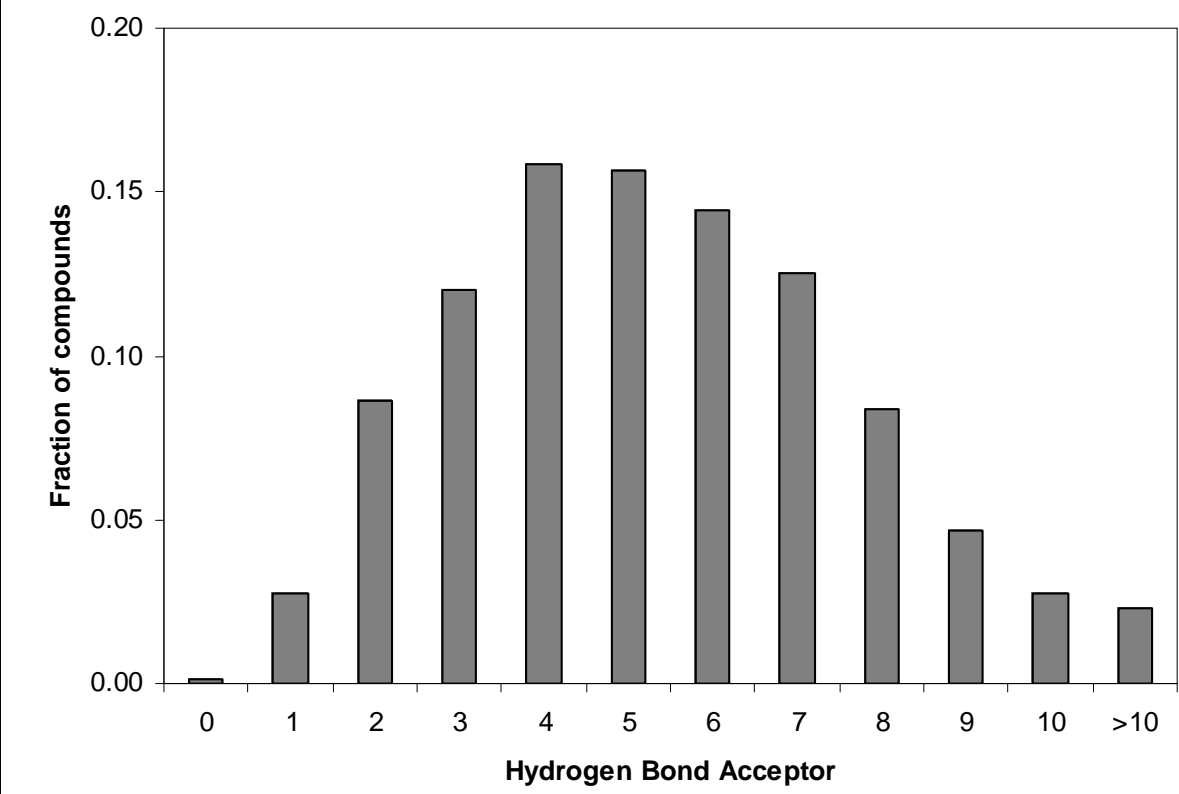




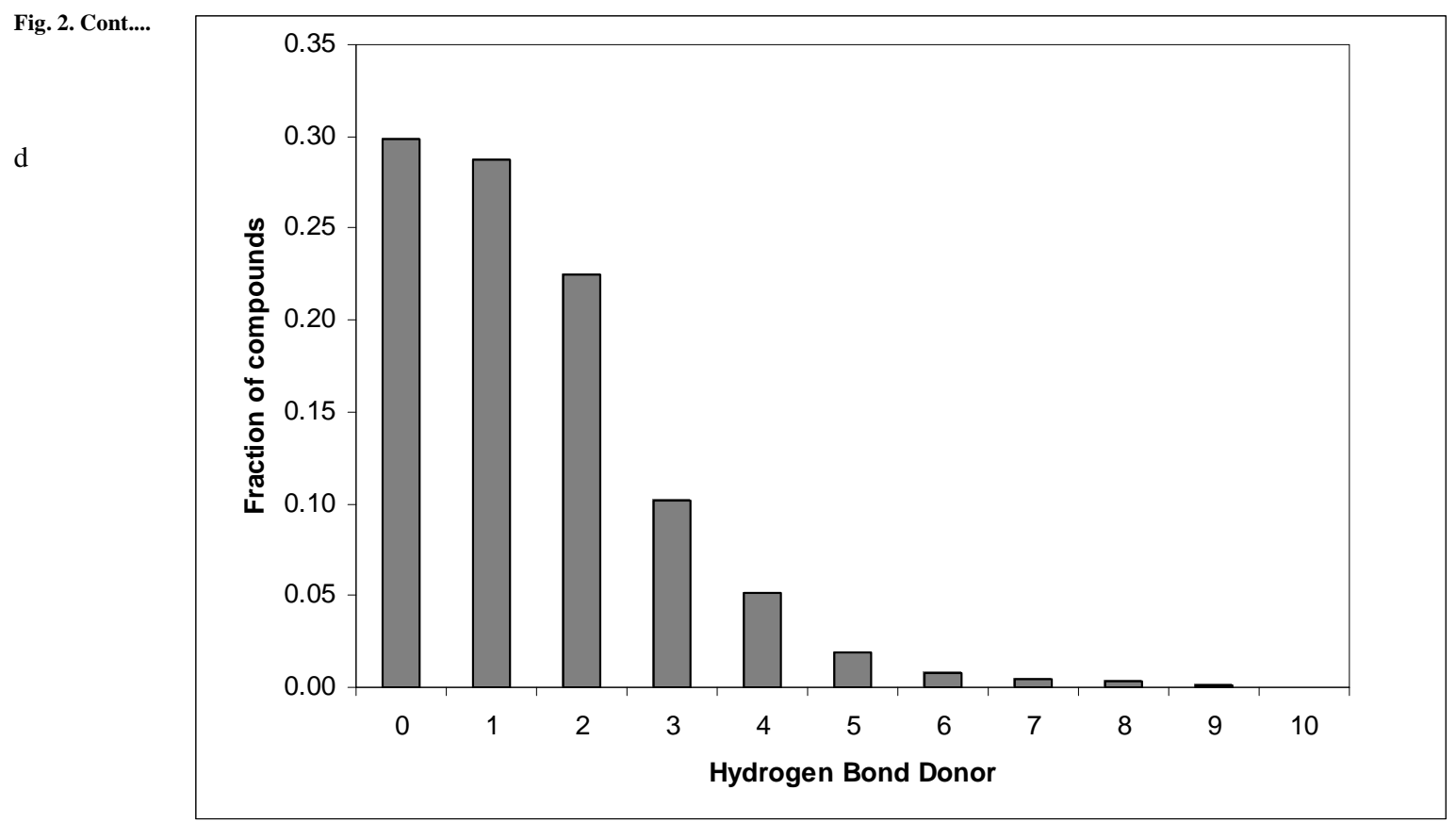

Fig. (2). Histograms of four physicochemical properties of compounds from the natural products database (NPD) showing a) molecular weight, b) $\log \mathrm{P}(\mathrm{o} / \mathrm{w}), \mathbf{c})$ hydrogen bond acceptors and $\mathbf{d})$ hydrogen bond donors. These selected descriptors are related to oral bioavailability and drug likeness. The physicochemical profile of the NPD molecules was computed by MOE 2008.10.

Table 2. Correlation Matrix for Natural Products

\begin{tabular}{|c|c|c|c|c|c|}
\hline Descriptor & KierFlex & Q_VSA_HYD & a_nN & chiral_u & 0.14 \\
\hline \hline KierFlex & 1 & 0.175 & -0.217 & 0.066 \\
\hline Q_VSA_HYD & 0.175 & 1 & -0.4 & -0.239 & -0.252 \\
\hline a_nN & -0.217 & -0.4 & -0.187 & -0.14 \\
\hline chiral_u & 0.14 & 0.066 & -0.14 & -0.038 & -0.038 \\
\hline vsa_acid & 0.187 & -0.252 & 1 \\
\hline
\end{tabular}

the correlation matrix of our model descriptors for the natural products sample contains only elements $\leq 0.4$ (in absolute values).

The property distribution of the proposed five discriminative descriptors for both the natural products database (NPD) and synthetic products database (CPD), are shown in Fig. (3). The histograms showing 3a) number of nitrogen atoms (a_nN), 3b) total hydrophobic Vander Waals area (Q_VSA_Hyd), 3c) number of unconstrained chiral centers (chiral_u), 3d) KierFlex - molecular flexibility index (KierFlex) and 3e) sum of VDW surface area of acidic atoms (vsa_acid). $x$ - axis label is the upper limit of binned data, e.g., 6 in KierFlex histogram is equivalent to 3-6. The enrichment factors (fraction of natural products/ fraction of synthetic chemicals) equal 5.8, 3.2, 5, 2.8 and 1.9 for a_nN=0; KierFlex $\leq 3$; chiral_u $\geq 3$; KierFlex $\leq 160$ and vsa_acid $>27$ respectively. Distribution histogram 3 a shows that number of nitrogen atoms peaks at 1 in natural products and at 2 in synthetic chemicals. As well, sum of VDW surface area of acidic atoms is higher in synthetic chemicals compared to natural products. As shown in Fig. (3b), it peaks at 220 in natural products and at 280 in synthetic chemicals.

Two thousands natural products and two thousands synthetic chemicals were selected randomly from the pool 5 times (assuring that the molecules in the new set have not been selected before for the previous sets). Table 3 lists the performance of the model on the different sets and as shown, the proposed model is highly robust.

Our natural likeness rules states that synthetic chemicals are more likely to have up to 1 violation while natural-based products having more than 1 violation to the following rules: a_nN $\geq 2,160 \leq$ Q_VSA_Hyd $\leq 548$, chiral_u $<3,3 \leq$ KierFlex $\leq 28$ and vsa_acid $\leq 27$. These extracted rules are useful for separating natural-based chemicals from synthetic ones. Mathews Correlation Coefficient of 0.5 is attained; nearly $81 \%$ of natural-based products and $68 \%$ of synthetic chemicals were correctly classified with this filter. In Fig. (4) we present some known natural-based drugs that are predicted as natural chemicals according to our proposed model. One 


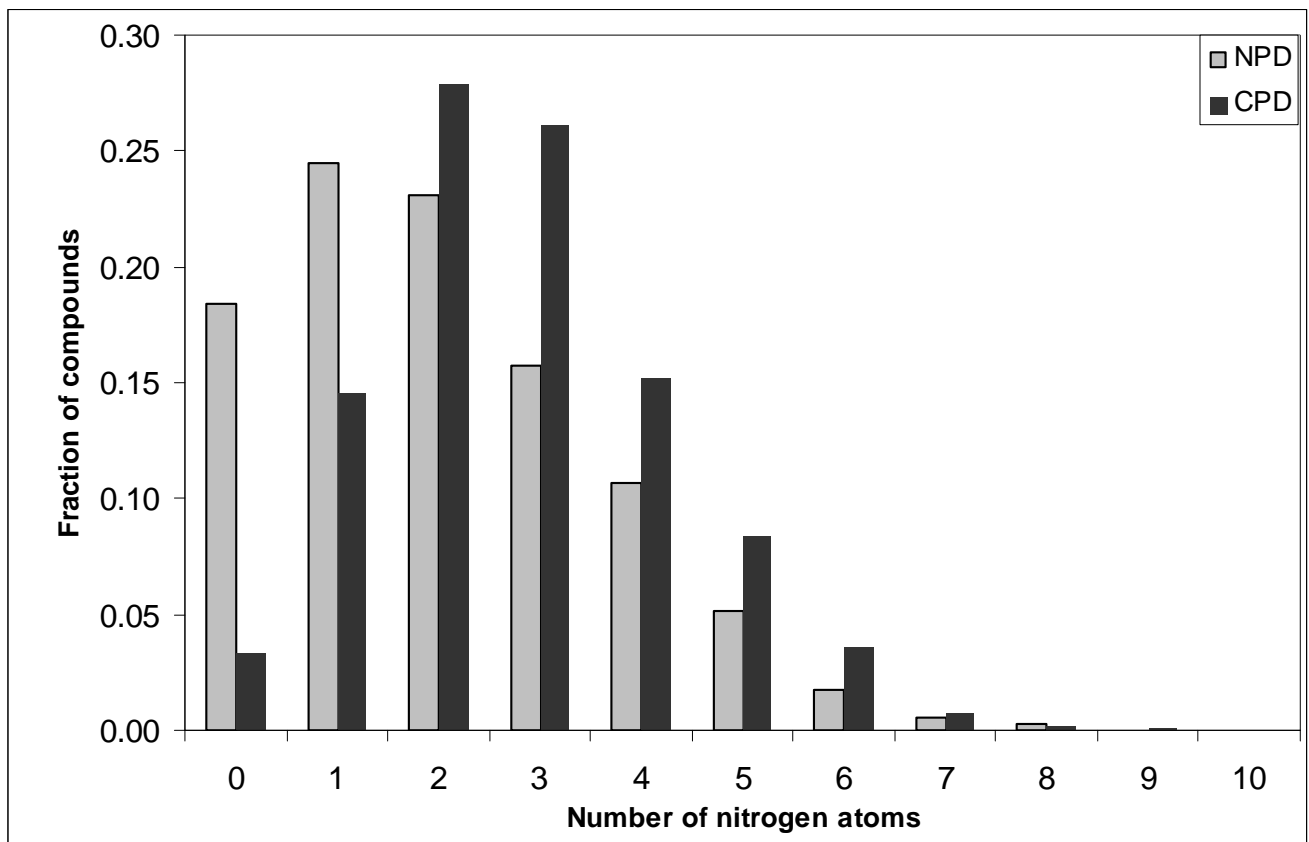

$\mathrm{b}$

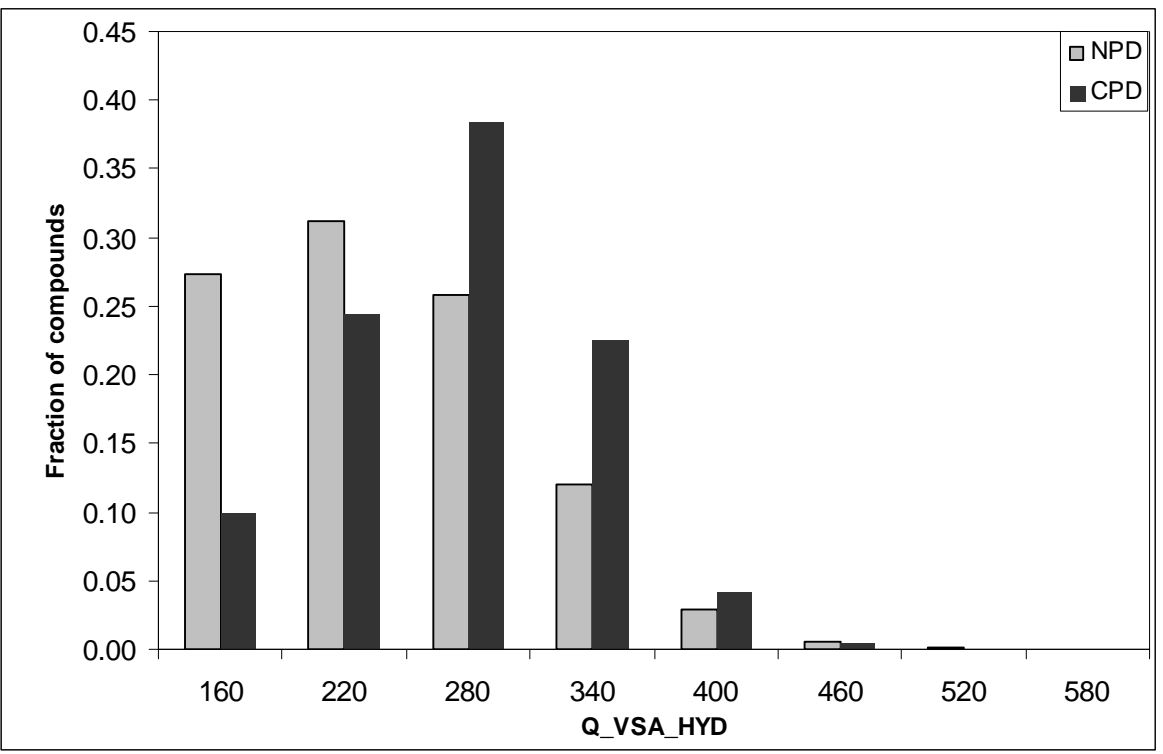

c

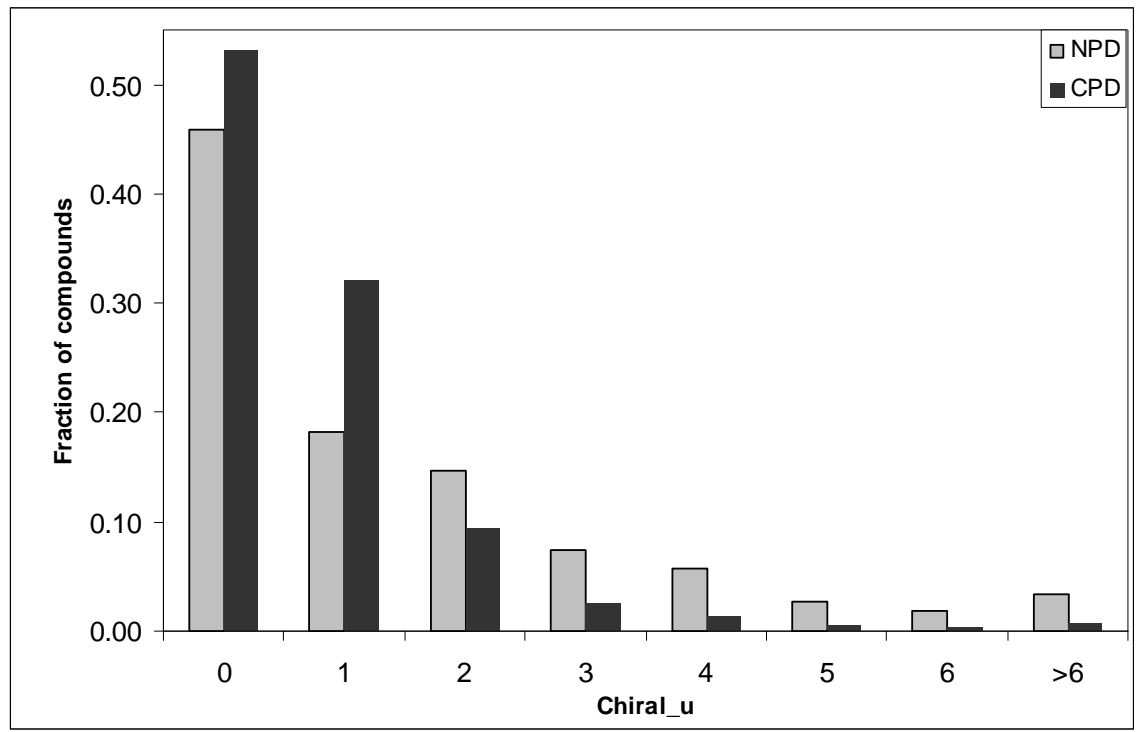


Fig. 3. Cont...

d

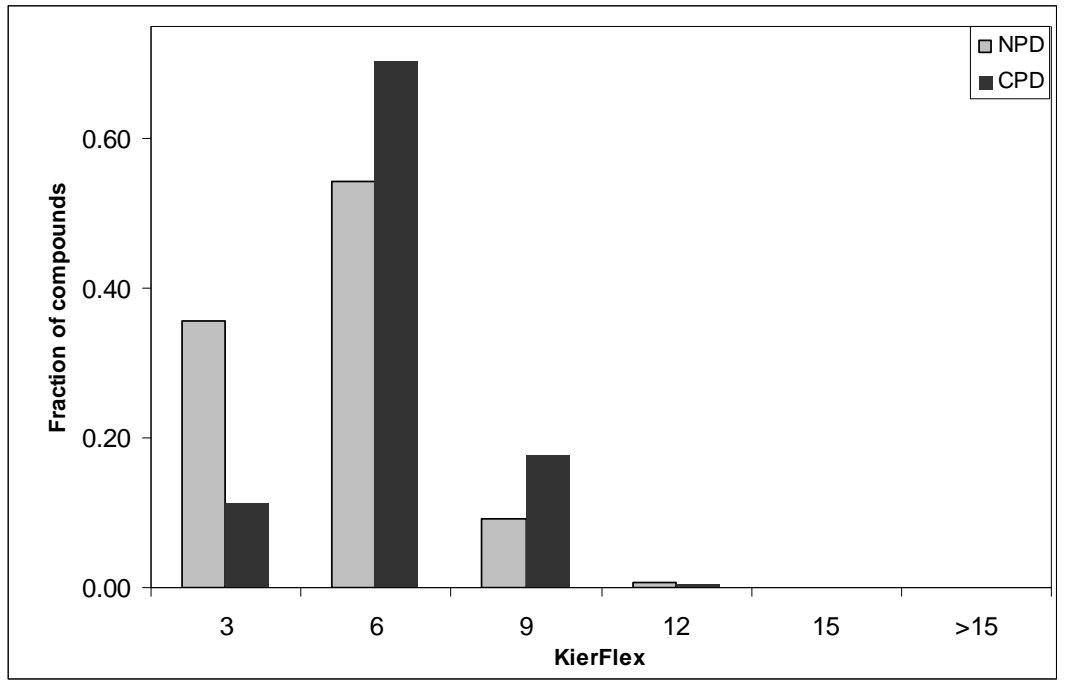

$\mathrm{e}$

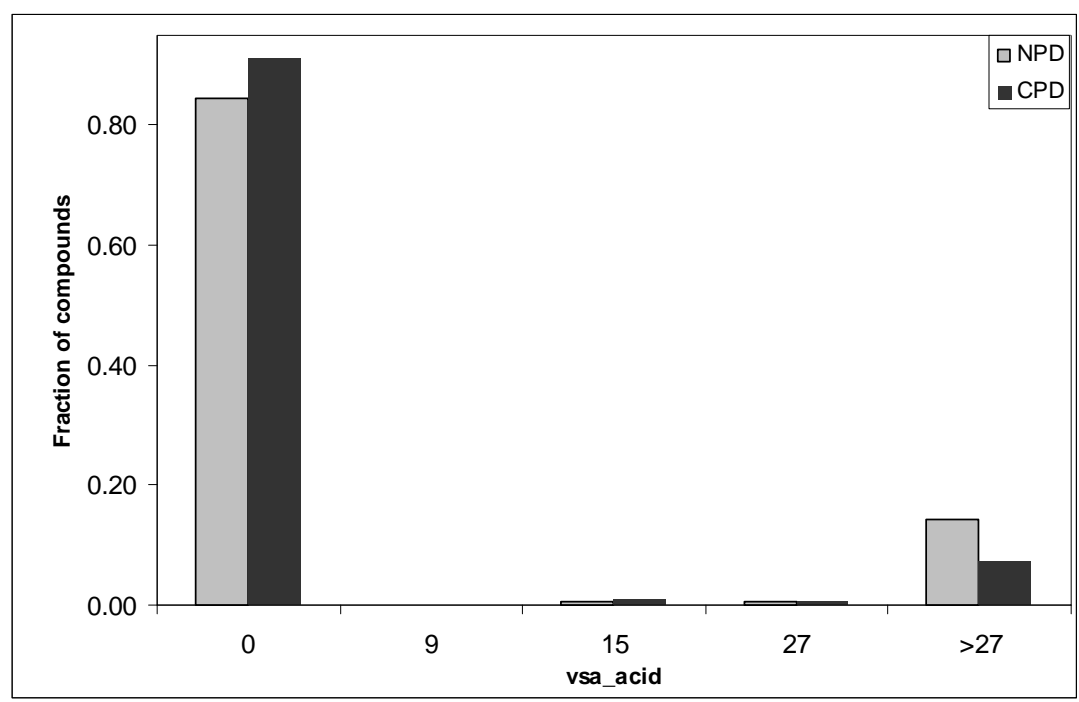

Fig. (3). Comparison of property distribution of five discriminative descriptors for the two data sets (NPD - natural products database, colored Gray and CPD - synthetic products database, colored black). The histograms showing a) number of nitrogen atoms, b) total hydrophobic Vander Waals area, c) number of unconstrained chiral centers, d) KierFlex - molecular flexibility index and e) sum of VDW surface area of acidic atoms. $x$ - axis label is the upper limit of binned data, e.g., 6 in KierFlex histogram is equivalent to 3-6.

Table 3. Robustness of the Proposed Model

\begin{tabular}{|c|c|c|c|}
\hline Partition Number & Natural\% & Synthetic\% & MCC \\
\hline \hline 1 & 80.8 & 68.1 & 0.5 \\
\hline 2 & 81.7 & 68.6 & 0.51 \\
\hline 3 & 80.5 & 67.3 & 0.49 \\
\hline 4 & 81.2 & 67.3 & 0.5 \\
\hline 5 & 80.7 & 68.3 & 0.5 \\
\hline
\end{tabular}

of the earliest success stories in developing a drug from a natural product was aspirin. It is chemically similar to Salicin which is a natural chemical produced from willow barks. Topiramate is a sulfamate derivative of the naturally occurring sugar D-fructose. Dydrogesterone molecular structure is almost identical to that of natural progesterone. Citric acid is a weak organic acid, and it is a natural chemical exists in a variety of fruits and vegetables. Tretinoin is the acid form of vitamin A. Pilocarpine is a parasympathomimetic alkaloid obtained from the leaves of tropical American shrubs from the genus Pilocarpus. Geraniol is a monoterpenoid and an alcohol considered as the primary part 

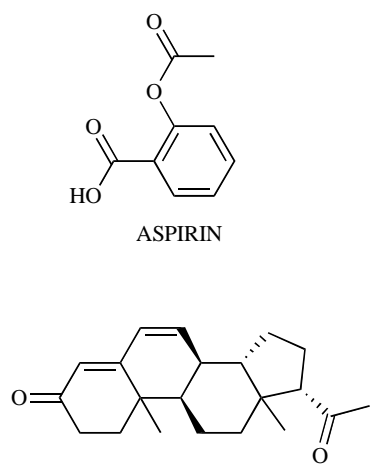

DYDROGESTERONE

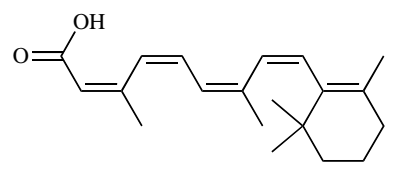

TRETINOIN

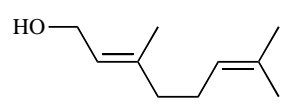

GERANIOL

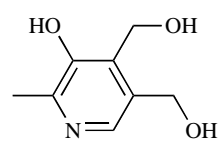

PYRIDOXINE
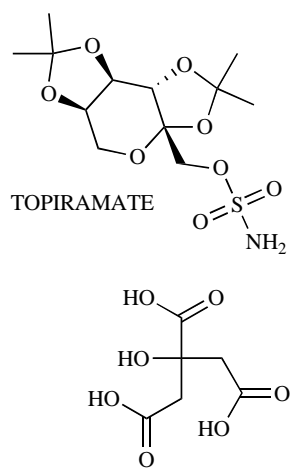

CITRIC ACID

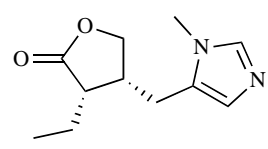

PILOCARPINE

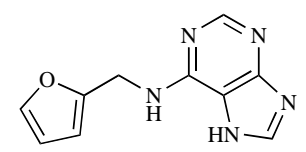

KINETIN

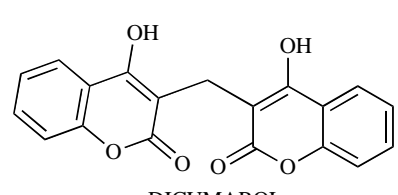

DICUMAROL
Fig. (4). Natural-based drugs.

of rose oil, palmarosa oil, and citronella oil. Kinetin is a kind of cytokinin, a class of plant hormone that promotes cell division. Pyridoxine is a vitamin B6 and the dragon fruit from South East Asia is a very good source for it. Dicoumarol is a natural chemical substance of combinded plant and fungal origin. It is a derivative of coumarin, a substance made by plants and transformed by a number of species of fungi into dicoumarol. Some of these drugs such as Aspirin, Pyridoxine and Dicumarol belong to the topselling prescribed drugs.

\section{CONCLUDING REMARKS}

A highly efficient rules based model for natural- likeness of chemicals has been unraveled and could be incorporated into virtual screening process of large chemical databases as well as in library design of natural like chemicals. As well, since natural based products are inherently better tolerated in the body than synthetic chemicals and have higher chance to be approved as new drugs, selection of natural-like drug candidates is justified. Our model for natural likeness proposed herein states that synthetic chemicals are more likely to have up to 1 violation while natural-based products having more than 1 violation to the following rules: Number of nitrogen atoms $\geq 2$, total hydrophobic Vander Waals area ranges between 160 and 548, number of unconstrained chiral centers is less than 3 , molecular flexibility index (KierFlex) ranges between 3 and 28, while sum of VDW surface area of acidic atoms is up to 27.
This model attained Mathews Correlation Coefficient of 0.5; nearly $81 \%$ of natural-based products and $68 \%$ of synthetic chemicals were correctly classified. The model is highly robust when was run on 5 different sets of natural products and synthetic chemicals.

\section{ACKNOWLEDGEMENT}

We gratefully acknowledge Dr. Mizied Falah for his comments on this manuscript.

\section{ABBREVIATIONS}

$\begin{array}{lll}\mathrm{NPD} & = & \text { Natural Products Database } \\ \mathrm{MCC} & = & \text { Matthews' Correlation Coefficient }\end{array}$

\section{REFERENCES}

[1] Lee KH. Discovery and Development of natural product-derived chemotherapeutic agents based on a medicinal chemistry approach. J Nat Prod 2010; 73(3): 500-16

[2] Rishton GM. Natural products as a robust source of new drugs and drug leads: past successes and present day issues. Am J Cardiol 2008; 101(10A): 43D-9D.

[3] Li JW, Vederas JC. Drug discovery and natural products: end of an era or an endless frontier? Science 2009; 325(5937): 161-5.

[4] Harvey AL. Natural products in drug discovery. Drug Discov Today 2008; 13(19-20): 894-901.

[5] McChesney JD, Venkataraman SK, Henri JT. Plant natural products: back to the future or into extinction? Phytochemistry 2007; 68(14): 2015-22.

[6] Tan G, Gyllenhaal C, Soejarto DD. Biodiversity as a source of anticancer drugs. Curr Drug Targets 2006; 7(3): 265-77.

[7] Hughes B. 2008 FDA drug approvals. Nat Rev Drug Discov 2009; 8(2): 93-6.

[8] Hughes B. 2007 FDA drug approvals: a year of flux. Nat Rev Drug Discov 2008; 7(2): 107-9.

[9] Harvey A. The role of natural products in drug discovery and development in the new millennium. IDrugs 2010; 13(2): 70-2.

[10] Beutler JA. Natural products as a foundation for drug discovery. Curr Protoc Pharmacol 2009; 46: 9.11.1.

[11] Ojima I. Modern natural products chemistry and drug discovery. J Med Chem 2008 8; 51(9): 2587-8.

[12] Newman DJ, Cragg GM. Natural products as sources of new drugs over the last 25 years. J Nat Prod 2007; 70(3): 461-77.

[13] Newman DJ, Cragg GM. Marine natural products and related compounds in clinical and advanced preclinical trials. J Nat Prod 2004; 67(8): 1216-38.

[14] Paterson I, Anderson EA. Chemistry. The renaissance of natural products as drug candidates. Science 2005; 310(5747): 451-3.

[15] Desai MC, Chackalamannil S. Rediscovering the role of natural products in drug discovery. Curr Opin Drug Discov Dev 2008; 11(4): 436-7.

[16] Chittchang M, Gleeson MP, Ploypradith P, Ruchirawat S. Assessing the drug-likeness of lamellarins, a marine-derived natural product class with diverse oncological activities. Eur J Med Chem 2010; 45(6): 2165-72.

[17] Breinbauer R, Vetter IR, Waldmann H. From protein domains to drug candidates-natural products as guiding principles in the design and synthesis of compound libraries. Angew Chem Int Ed Engl 2002; 41(16): 2879-90.

[18] Balamurugan R, Dekker FJ, Waldmann H. Design of compound libraries based on natural product scaffolds and protein structure similarity clustering (PSSC). Mol Biosyst 2005; 1(1): 36-45.

[19] Kong DX, Ren W, Lu W, Zhang HY. Do biologically relevant compounds have more chance to be drugs? J Chem Inf Model 2009; 49(10): 2376-81.

[20] Vijayakrishnan R. Structure-based drug design and modern medicine. J Postgrad Med 2009; 55(4): 301-4.

[21] Rayan A, Noy E, Chema D, Levitzki A, Goldblum A. Stochastic algorithm for kinase homology model construction. Curr Med Chem 2004; 11(6): 675-92.

[22] Jorgensen WL. Efficient drug lead discovery and optimization. Acc Chem Res 2009; 42(6): 724-33. 
[23] Rollinger JM, Langer T, Stuppner H. Integrated in silico tools for exploiting the natural products' bioactivity. Planta Med 2006; 72(8): 671-8.

[24] Avila CM, Romeiro NC, Sant'Anna CM, Barreiro EJ, Fraga CA. Structural insights into IKKbeta inhibition by natural products staurosporine and quercetin. Bioorg Med Chem Lett 2009; 19(24): 6907-10.

[25] Ogungbe IV, Setzer WN. Comparative molecular docking of antitrypanosomal natural products into multiple Trypanosoma brucei drug targets. Molecules 2009; 14(4): 1513-36.

[26] Liu H, Li Y, Song M, et al. Structure-based discovery of potassium channel blockers from natural products: virtual screening and electrophysiological assay testing. Chem Biol 2003; 10(11): 110313 .

[27] Rollinger JM, Steindl TM, Schuster D, et al. Structure-based virtual screening for the discovery of natural inhibitors for human rhinovirus coat protein. J Med Chem 2008; 51(4): 842-51.

[28] Tanrikulu Y, Rau O, Schwarz O, et al. Structure-based pharmacophore screening for natural-product-derived PPARgamma agonists. ChemBioChem 2009; 10(1): 75-8.

[29] Brogi S, Kladi M, Vagias C, Papazafiri P, Roussis V, Tafi A. Pharmacophore modeling for qualitative prediction of antiestrogenic activity. J Chem Inf Model 2009; 49(11): 2489-97.

[30] Rollinger JM, Langer T, Stuppner H. Strategies for efficient lead structure discovery from natural products. Curr Med Chem 2006; 13(13): 1491-507.

[31] Franke L, Schwarz O, Muller-Kuhrt L, et al. Identification of natural-product-derived inhibitors of 5-lipoxygenase activity by ligandbased virtual screening. J Med Chem 2007; 50(11): 2640-6.

[32] Rollinger JM, Stuppner H, Langer T. Virtual screening for the discovery of bioactive natural products. Prog Drug Res 2008; 65(211): 3-49

[33] Shen J, Xu X, Cheng F, et al. Virtual screening on natural products for discovering active compounds and target information. Curr Med Chem 2003; 10(21): 2327-42.
[34] Cheng Y, Wang Y, Wang X. A causal relationship discovery-based approach to identifying active components of herbal medicine. Comput Biol Chem 2006; 30(2): 148-54.

[35] Koch MA, Schuffenhauer A, Scheck M, et al. Charting biologically relevant chemical space: a structural classification of natural products (SCONP). Proc Natl Acad Sci USA 2005; 102(48): 17272-7.

[36] Lee ML, Schneider G. Scaffold architecture and pharmacophoric properties of natural products and trade drugs: application in the design of natural product-based combinatorial libraries. J Comb Chem 2001; 3(3): 284-9.

[37] Stahura FL, Godden JW, Xue L, Bajorath J. Distinguishing between natural products and synthetic molecules by descriptor Shannon entropy analysis and binary QSAR calculations. J Chem Inf Comput Sci 2000; 40(5): 1245-52.

[38] Ertl P, Roggo S, Schuffenhauer A. Natural product-likeness score and its application for prioritization of compound libraries. J Chem Inf Model 2008; 48(1): 68-74.

[39] Rayan A, Goldblum A. Stochastic method to determine, in silico, the drug-like character of molecules. United States Patent Application 200701563432007.

[40] Rayan A, Marcus D, Goldblum A. Predicting Oral Druglikeness by Iterative Stochastic Elimination. J Chem Inf Model 2010; 50(3): 437-45.

[41] Frimurer TM, Bywater R, Naerum L, Lauritsen LN, Brunak S. Improving the odds in discriminating "Drug-like" from "Non Drug-like" compounds. J Chem Inf Comput Sci 2000; 40(6): 131524.

[42] Lipinski CA, Lombardo F, Dominy BW, Feeney PJ. Experimental and computational approaches to estimate solubility and permeability in drug discovery and development settings. Adv Drug Deliv Rev 2001; 46(1-3): 3-26.

[43] Oprea TI, Gottfries J. Toward minimalistic modeling of oral drug absorption. J Mol Graph Model 1999; 17(5-6): 261-74, 329. 